\title{
Structure of vitreous body and its relationship with liquefaction
}

\author{
Makoto Kodama ${ }^{1,2 *}$, Toyoaki Matsuura ${ }^{2}$, Yoshiaki Hara ${ }^{2}$ \\ ${ }^{1}$ Graduate School of Life Science and Systems Engineering, Kyushu Institute of Technology, Fukuoka, Japan \\ ${ }^{2}$ Department of Ophthalmology, Nara Medical University, Nara, Japan \\ Email: ${ }^{k}$ kodama@vessels.co.jp
}

Received 28 April 2013; revised 30 May 2013; accepted 10 June 2013

Copyright (C) 2013 Makoto Kodama et al. This is an open access article distributed under the Creative Commons Attribution License, which permits unrestricted use, distribution, and reproduction in any medium, provided the original work is properly cited.

\begin{abstract}
The aim of the study is to clarify the vitreous body structure and liquefaction phenomena. It was found that when melting a frozen rabbit vitreous body, the gel-sol transition phenomenon occurs and the gel structure is broken. This is almost like the liquefaction of the vitreous body in vivo. We try to clarify the liquefaction phenomenon by using this animal model. The native vitreous body has three dimensional meshwork structures. After liquefaction, it is changed into two parts, namely fiber aggregates and soluble amorphous aggregates. The surface of native vitreous body meshwork is mucopolysacharide, but that of fiber aggregates after liquefaction is changed into connective tissue, which means the conformational change of vitreous body in liquefaction. The soluble proteins after liquefaction were analyzed and identified as crystallin family. It is suggested that the liquefaction is induced by detachment of non-collagenous protein beads containing crystallins, resulting in the collapse of the three dimensional structure to release watery liquid trapped within. And the new gel-sol transition model of vitreous bod is proposed.
\end{abstract}

Keywords: Vitreous Body; Liquefaction; Crystallin

\section{INTRODUCTION}

The vitreous body is a transparent matrix that fills the posterior cavity of the eye, occupying more than two thirds of the intraocular volume. The mammalian vitreous body is a hydrophilic gel with a water content higher than $98 \%$. As Sebag [1] pointed out, the most enigmatic of ocular structures, vitreous has been unappreciated with respect to its role in health and disease, as compared

*Corresponding author. The present address: Vessel Inc., Fukuoka, Japan. with lens or retina.

Due to the highly viscous hydrogel, the precise structure of the vitreous body, including the mechanism of suppressing cell growth, has not yet been clarified, although some fibrous structures have been identified [2,3]. The vitreous body is composed of a sparse, random meshwork of collagen fibrils and that HA (hyarulonic acid) is dissolved in the interstices of the collagen fibrils without chemical links to the collagen. It has been thought that polymeric HA may adopt a random coil configuration and that the high viscosity of HA in solution is due to entanglement. But, a vitreous liquefaction phenomenon of phase change from gel to sol cannot be explained by this model. Recently, the vitreous body of most mammals has been shown to contain three polymeric components, namely, collagen, HA, and beaded fibrils $[4,5]$. However, the composition and function of these beads have remained unknown. The beads [6] may serve to stabilize the three-dimensional structure of vitreous body.

With aging, there is a localized breakdown of the gel structure in the human vitreous body with aggregation of the fibrils [7], which appears to be a frequent precursor to retinal detachment, resulting in liquefaction. The liquefaction of the vitreous body is the most common degenerative change occurring in senile conditions, which sometimes cause damage to the eyesight. So it is essential to obtain good and easy in vitro model of liquefaction to clarify the phenomenum.

When the frozen $\left(-20^{\circ} \mathrm{C}\right)$ vitreous body of rabbit is melted at the room temperature, it is separated into two phases, namely, the watery sol part, and the more tight gel, almost like the in vivo liquefaction, which is thought to be good in vitro model of liquefaction [8]. We try to clarify the structure of the vitreous body to make clear the mechanism of liquefaction using this model. 


\section{MATERIALS AND METHODS}

\subsection{Animals}

Male Japanese White Rabbits, 8 to 10 months of age (mean weight $3 \mathrm{~kg}$ ), were purchased from Kyudo Co. Ltd. (Fukuka, Japan) and kept under clean conditions at Kyushu Institute of Technology. All experiments were conducted in compliance with the ARVO Statement for the Use of Animals in Ophthalmic and Vision Research. The study was approved by the Animal Experiment Review Board of Graduate School of Life Science and Systems Engineering, Kyushu Institute of Technology.

\subsection{Preparation of Specimen for Microscopic Observation}

The rabbits were sacrificed by excess extracorporeal injection of pentobarbital. Within 1 hour, the rabbits' eye balls were enucleated, and first prefixed with $2 \mathrm{M}$ glutaraldehyde solution for $20 \mathrm{~min}$ and small incisions were made on the outer half-fixed surface of the eyeball, which reached to the vitreous humor near the nerve to allow entry of fixing reagents. Eyes were further fixed with $2 \mathrm{M}$ glutaraldehyde solution for $5 \mathrm{~min}$, and then with $10 \%$ formaldehyde in phosphate buffer $(\mathrm{pH} 7.5)$ for 1 day. This method is essentially the same as Kobayashi's method [9].

The samples were stained by the following methods, the phosphomolibdenic acid hematoxylin stain [10] and the double stain with Alcian Blue [11] and van Gieson [12]. They were examined on a Leica Microscope LMD at visible or dark phase.

\subsection{Two-Dimensional Gel Electrophoresis}

In order to obtain the native vitreous sample, rabbit vitreous humor was aspirated from central vitreous within 1 hour after death. In order to prepare the melted vitreous sample, rabbit eye was frozen at $-20^{\circ} \mathrm{C}$. The frozen vitreous was dissected from the eye ball and was left for 30 minutes at $4{ }^{\circ} \mathrm{C}$ to melt the frozen vitreous. After melting, the soluble portion which released from the frozen vitreous was used as the melted vitreous sample.

The native and the melted vitreous samples were analyzed by two-dimensional electrophoresis (isoelectric focusing electrophoresis with $8 \mathrm{M}$ urea condition and SDS-PAGE) as described previously [13]. Two-dimensional gel electrophoresis was performed using immobilized pH gradient stripes, pH3-10 (Protean IEF cell; Bio$\mathrm{Rad})$. Proteins were resolved by isoelectric focusing in the first dimension and SDS-Page (12\% acrylamide) in the second dimension. Protein spots were visualized by $0.125 \%$ Coomassie brilliant blue R-250 in $25 \%$ ethanol staining overnight and then destained with the solution of $25 \%$ ethanol and $0.35 \%$ formaldehyde. They were iden- tified based on their discrete presence in the gel.

\subsection{In-Gel Tryptic Digestion}

The selected spots of proteins were excised manually from gels and were subjected to in-gel digestion using sequencing grade bovine trypsin (Sigma) reconstituted in 25 $\mathrm{mM}$ ammonium bicarbonate at $\mathrm{pH}$ 8.0. After digestion, peptides from the gel were extracted twice using $100 \mu$ of $50 \%$ acetonitrile containing $5 \%$ trifluoroacetic acid.

\subsection{Mass Spectrometry and PMF (Peptide Mass Fingerprints) Analysis}

Peptide mass fingerprinting of the reconstituted peptides was obtained using the Reflection model of a time-offlight MALDI-TOF MS AXIMA-CFR (Shimadzu Corporation at Tsukuba City) with delayed extraction. Spetra were obtained by the summation of laser spots. Searches with Mascot (Matric Science Ltd., UK), in the NCBI data base were performed to identify amino acid sequence with a mass tolerance of $100 \mathrm{ppm}$.

\section{RESULTS}

\subsection{Cross Sectional Views of Vitreous Body}

Three dimensional meshwork structures are observed in rabbit native vitreous body near retina and at most joints of the fibril meshwork, bead-like joints are observed as shown in Figure 1. This meshwork structure is homogeneous and observed at whole vitreous body of a native rabbit eye. The circled parts of Figures 1(a) and (b) are magnified to Figures 1(c) and (d), respectively. These more magnified pictures (c) and (d) show clearly the presence of beads at meshwork structure, which seems to be located at the joint of the meshwork. This meshwork structure is more clearly observed at dark phase as Figures 1(b) and (d) show, rather than at bright phase as Figures 1(a) and (c) show. Especially, the dark phase picture in Figure 1(d) reveals shining bead-like joints of the meshwork as the arrowheads indicate. When the frozen vitreous body $\left(-20^{\circ} \mathrm{C}\right)$ is melted, the vitreous body is changed into two phases, that is, associated fibrils (the arrowheads in Figures 2(a) and (b)) and amorphous aggregates (the arrowheads in Figures 2(c) and (d)). The beads are not detected at the joints of the associated fibrils of the melted vitreous body in Figures 2(a) and (b). After the melted vitreous body was rinsed with saline, the amorphous aggregates (the arrowhead in Figures 2(c) and (d)) were not observed, except fiber aggregates as observed in Figures 2(a) and (b). The amorphous aggregates in Figures 2(c) and (d) (the arrowhead) are soluble and seem to be released from the associated fibrils in Figures 2(a) and (b).

The double stain with Alcian Blue and van Gieson re- 


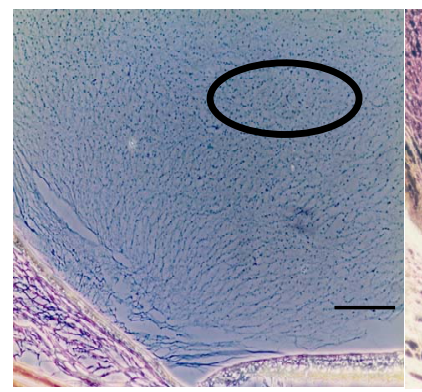

(a)

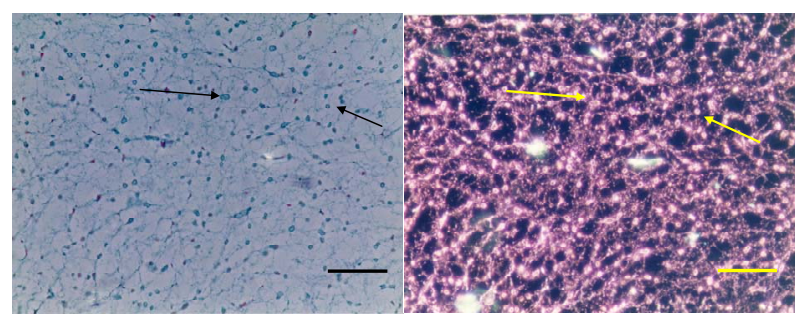

(c)

(d)

Figure 1. Cross-sectional views of a native and melted eye of rabbit dark phase image (bars $50 \mu \mathrm{m}$ ) by phosphomolibdenic acid hematoxylin stain. (a) Bright phase view; (b) Dark phase view; (c) Bright phase view; (d) Dark phase view.

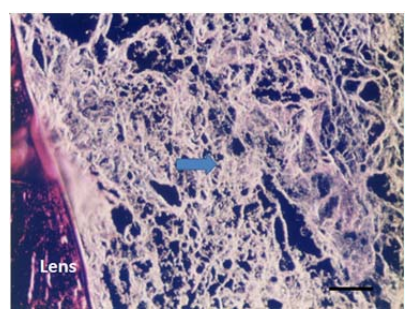

(a)

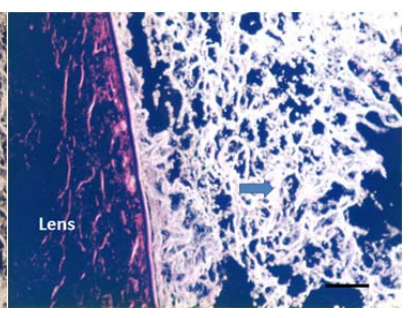

(b)

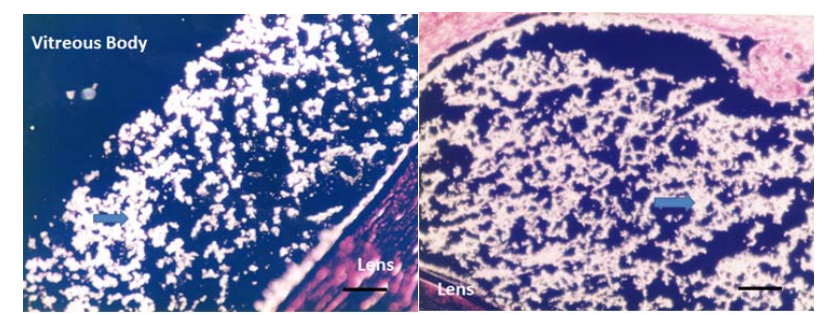

(c)

(d)
Figure 2. Cross-sectional views of the Melted Vitreous Body of Rabbit by dark phase image (Magnification. bars: $50 \mu \mathrm{m}$ ) by Phosphomolibdenic acid-Hematoxylin eosin stain. (a) Fibrous Aggregates-1; (b) Fibrous Aggregates-2; (c) Amorphous Aggregates-1; (d) Amorphous Aggregates-2.

veals the conformational changes of the melted vitreous body as shown in Figures 3(a) and (b). Homogeneous and 3 dimensional networks with bead (arrowhead in Figure 3(a)), stained blue (positive in Alcian Blue), are observed in the native vitreous body. However, in the melted (liquefied) vitreous body there are mainly red stained fiber aggregates without any bead (arrowhead in Figure 3(b)) and minor blue stained fibers (arrowhead in Figure 3(b)).

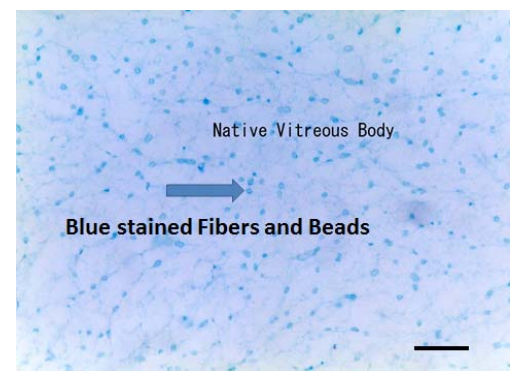

(a)

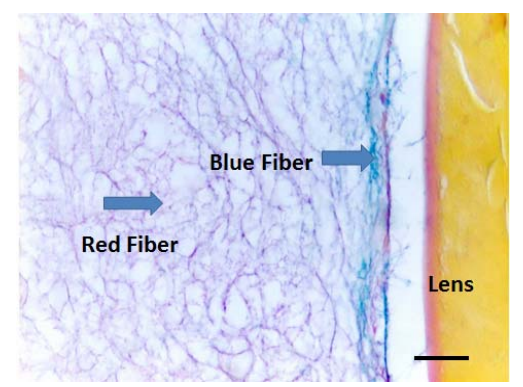

(b)

Figure 3. Cross-sectional views of the Native and Melted (Liquefied) Vitreous Body of Rabbit by the double stain with Alcian blue and van Gieson (Magnification. bars: $50 \mu \mathrm{m}$ ) (a) Native Vitreous Body; (b) Melted (Liquefied) Vitreous Body.

\subsection{Two Dimensional Gel Electrophoresis of Vitreous body and Lens}

The two-dimensional gel electrophoresis patterns of the soluble proteins from native and melted vitreous bodies of a rabbit are shown in Figure 4. There are not any significant spots in Figure 4(a) of the native vitreous body. On the other hand, the melted vitreous body (Figure 4(b)) shows many spots in the molecular weight range of 20 and $32 \mathrm{kDa}$ and the $\mathrm{pH}$ range from 5 to 6.4 . This distribution of major spots of melted vitreous body (Figure 4(b)) are quite similar to that of the spots of the lens soluble proteins (Figure 4(c))

\subsection{Analysis of MALDI-TOF MS of Vitreous Body and Lens}

We took one of the major spots, ( $\alpha$ spot in Figures 4(b) and (c)) of a rabbit of almost same molecular mass and same pI value and analyzed by MALDI-TOF MS. The both of spots (in Figures 4(b) and in 4(c)) were identified as $\alpha$ A-crstallin at Table 1.

\section{DISCUSSION}

\subsection{Structure of Vitreous Body-3 Dimentional Net Work Structure}

Three dimensional meshwork structure of a rabbit native 
Table 1. Mascot seach results.

\begin{tabular}{|c|c|}
\hline & $\begin{array}{l}\text { Proteins Identified in Vitreous Body and } \\
\text { Lens of Rabbit Mascot Search Results }\end{array}$ \\
\hline & $\begin{array}{l}\text { One spot } \alpha \text { denoted in Figure } 4(\mathbf{b}) \text { in the liquefied rabbit } \\
\text { vitreous body (Matched amino acids shown in bold italic) }\end{array}$ \\
\hline 1 & $\begin{array}{l}\text { MDVTIQHPWF KRTLGFYPS RLFDQFFGEG } \\
\text { LFEYDLLPFL SSTISPYYQ }\end{array}$ \\
\hline 51 & $\begin{array}{l}\text { SLFRTVLDSG ISEVRSDRDK FVIFLDVKHF } \\
\text { SPEDLTVKVQ EDFVEIHGKH }\end{array}$ \\
\hline 101 & $\begin{array}{c}\text { NERQDDHGYI SREFHRRYRL PSNVDQSALS } \\
\text { CSLSADGMLT FSGPKVQSGL }\end{array}$ \\
\hline \multirow[t]{2}{*}{151} & DADHSERAIP VSREEKPSSA PSS \\
\hline & $\begin{array}{l}\text { One spot } \alpha \text { denoted in Figure 4(c) in the rabbit lens } \\
\text { (Matched amino acids shown in bold italic) }\end{array}$ \\
\hline 1 & $\begin{array}{l}\text { MDVTIQHPWF KRTLGFYPS RLFDQFFGEG } \\
\text { LFEYDLLPFL SSTISPYYQ }\end{array}$ \\
\hline 51 & $\begin{array}{l}\text { SLFRTVLDSG ISEVRSDRDK FVIFLDVKHF } \\
\text { SPEDLTVKVQ EDFVEIHGKH }\end{array}$ \\
\hline 101 & $\begin{array}{l}\text { NERQDDHGYI SREFHRRYRL PSNVDQSALS } \\
\text { CSLSADGMLT FSGPKVQSGL }\end{array}$ \\
\hline 151 & DADHSERAIP VSREEKPSSA PSS \\
\hline
\end{tabular}

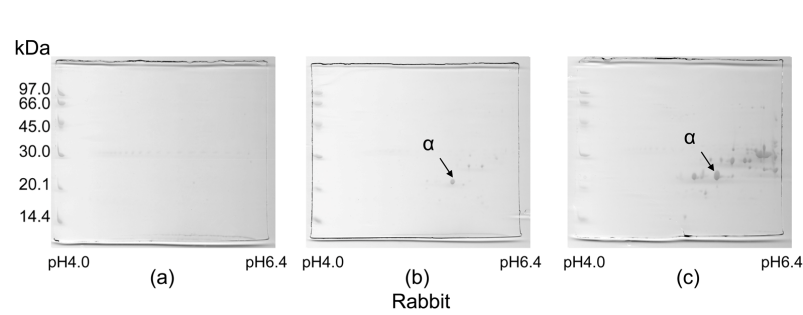

Figure 4. Two-dimensional gel electrophoresis patterns of soluble proteins obtained from native and melted vitreous bodies and from lens of rabbits. (a) Soluble proteins of the native vitreous body; (b) Soluble proteins of the melted vitreous body; (c) Soluble proteins of the lens.

vitreous body in Figures 1(c) and (d) could not be clearly detected with hematoxylene eosin stain or Azan stain. It may due to the surface chemical structure of HA (hyaluronic acid) in the vitreous body. Figure 3(a) also shows the existence of mucopolysacharide which is Alcian blue positive in the mesh work structure of vitreous body. Bead-like substances of $2-4 \mu \mathrm{m}$ in diameter are present at most joints of the fibril meshwork as shown by the arrowhead in Figures 1 and 3(a). These beads are clearly observed at dark phase view as the arrowheads in Figure 1(d). They are shining and clearly distinguished from the fibrils as compared with the arrowheads $\alpha$ in Figure 1(c). These shining beads mean the different components from the meshwork fibers.

After melting from the frozen state, the vitreous body structure is changed into the two phases. One phase is associated fibrils as the arrowheads in Figures 2(a) and (b) show, which do not have the beads. Another phase is amorphous aggregates as the arrowheads in Figures 2(c), (d) show. After rinsing the melted vitreous body with saline or distilled water, the amorphous aggregates as the arrowhead in Figures 2(c) and (d) were not observed except the associated fibrils as the arrowhead in Figures 2(a) and (b). These amorphous aggregates are water-soluble and thought to be derived from beads observed in Figure 1.

The double stain method reveals more precisely the conformational fibers change of vitreous body in Figure 3. Native vitreous body shows homogeneous blue stained 3 dimensional networks with beads as Figure 1 shows. It means the surface of native vitreous body fiber is glucosamine (probably hyaluronic acid). However, the melted vitreous body shows mainly red stained fiber aggregates, which means connective tissues (probably collagen) without any beads. These results are coincident with those of Figures 1 and 2. It was found [13] that ruthenium red positive glycosaminoglycans exist as amorphous masses on collagen fibrils, as well as filaments intercomnecting collagen fibrils and amorphous materials. It is proposed that HA binds with collagen fibrils via chondroitin sulfate.

This meshwork structure can hold so much watery liquid in vitreous body to form gel-like structure. As Scott pointed out [14], the water of the vitreous is stabilized by an orderd meshwork of very fine collagen fibrils that are tied in loosely parallel bundles or sprays by bidges or sulphated glycosaminoglyxan. The protein cores of the proteoglycans are attached to collagen fibrils. Thus, Collagen fibrils-proteoglycan protein core glycosaminoglycan chain.

\subsection{The Mechanism of Liquefaction of Vitreous Body}

What is the mechanism of liquefaction? Liquefaction is a sort of phase transition from gel phase to sol phase. If water-like aqueous humor is merely trapped as binding waters of hyaluronic acid in vitreous body [3], this phase transition is not easily elucidated.

Bishop [15] hypothesized that synchisis and syneresis may be caused by the gradual loss of proteoglycan coating from collagen fibrils that are seen with aging. Sticky collagen fibrils would then be able to contact each other and aggregate. Collagen degradation [16,17] or fibril formation $[18,19]$ makes an important role in liquefaction. Breakdown of collagen fibrils into smaller fragments [20] is thought to be crucial to the age-related liquefaction of the human vitreous body. Interleukin 113 [21] and light [22] are also the potent factors to induce this liquefaction.

It was suggested [23] that the gel vitreous can be liquefied by removing or destroying the collagen fibrillar network. The former can be achieved by filtration or by centrifugation, the latter by homogenization or by digestion with proteases (collagenases) which destroy the fibrillar collagen. The residue after filtration or the sedi- 
ment after centrifugation was called "residual protein". In this experiment, the residual protein itself must be beads proteins.

On the other hands, Worst [5] found a characteristic fine structure in vitreous consisting of a number of radially and concentric running wavy lines, in a fairly complex arrangement. It was revealed by the transmission electron micrographs [24] that some collagen fibrils in human vitreous appeared to be packed in a bundle of parallel fibrils to form big fibrils of diameter $10-20 \mu \mathrm{m}$. And the existence of amorphous beads like substance of diameter 10 - $40 \mu \mathrm{m}$ were also observed.

It is suggested from this experimental results that these break down trigger may induce detachment of beads protein from the joint of meshwork structure. The gel vitreous [25] can be liquefied by removing or destroying the collagen fibrillar network. There are research works $[4,6,14]$ which show very small fibrils and beads by transmission electron micrograph. But, as Ponsioen [26] pointed out, it is uncertain that morphological changes in the aging vitreous, liquefaction, is due to a simple aggregation of collagen fibrils. The all components of vireous body must be related with liquefaction.

If aqueous is trapped with three dimensional meshwork structure of HA (hyaluronic acid)-collagen fibrils as shown in this study, this aqueous is easily released from the meshwork after this structure is broke down.

It is suggested from these results that the three dimensional meshwork can hold watery liquid and form stable gel network. However, the meshwork is broken down by detaching beads at the joint of meshwork, which may reinforce the structure, resulting the drastic conformational change of meshwork fiber and the release this watery liquid from the meshwork. This process is explained later using the models in Figures 5 and 6.

\subsection{Existence of Crystallin in Vitreous Body}

From the results of two dimensional gel electrophoresis, it is also revealed that these soluble proteins (Figure 4(b)) were released from the meshwork structure after being melted. It must be pointed out that the spots of the two dimensional gel electrophoresis in Figure $\mathbf{4}$ are obtained without any concentration procedure of the sample soluble proteins solution [13]. Where do these protein spots come from? Any major proteins are not detected in Figure 4(a) of native vitreous body. These spots must be identical with the amorphous aggregates in Figures 2(c) and (d). Before liquefaction, any significant proteins were not detected as in Figure 4(a) of a rabbit.

We selected one of the major spots, $\alpha$ spot in Figures 4(a) and (c), respectively, of molecular mass between 20 and $25 \mathrm{kDa}$ and analyzed by MALDI-TOF MS as Table 1 shows. The sequences of spot $\alpha$ in Figure 3(b) and spot $\alpha$ in Figure 3(c) were consistent with the result

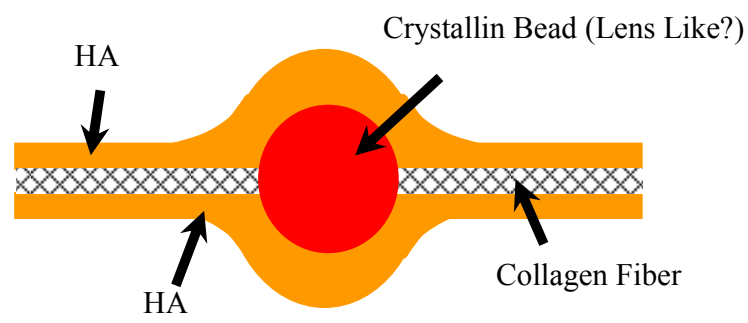

Figure 5. The model for fiber of vitreous body meshwork structure.

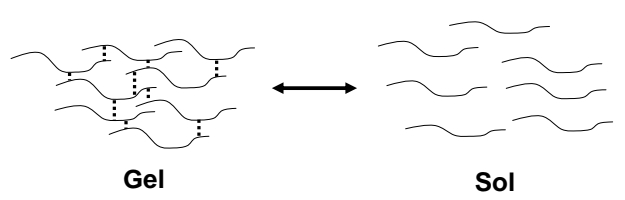

(a)

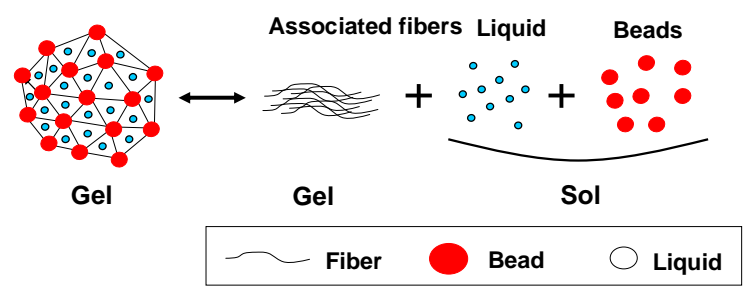

(b)

Figure 6. The model for gel-sol transition of vitreous body liquefaction. (a) Polymer gel-sol transition model; (b) Vitreous gel-sol transition model.

(V146-QSGLDAGH) of the $\alpha \mathrm{A}$-crystallin fragment of lens [14] at Table 1. These spots $\alpha$ are identified as a $\alpha \mathrm{A}$-crystallin (Table 1). $\alpha \mathrm{A}$-crystallin is one of the main components of the lens [26,27] and a lens-specific protein, even though it is expressed in non-ocular tissues [28]. There are some papers revealing the existence of different type of crystallins, suggesting that there is a relationship between the lens and the vitreous body.

Other spots in Figure 3(b) of a rabbit are also identified as crystallin family by the same Mascot Analysis (data are not shown).

There have been many proteome studies about vitreous body [29-36]. Other type crystallins were also found in vitreous [18,20-24]. The distribution of spots in Figure 4(b) of the melted vitreous body and Figure 4(c) of the lens soluble proteins are similar each other, suggesting they are crystallin family as Table $\mathbf{1}$. This result is consistent with Davidson's result [4], which also shows non-collagenous substance of beads. $\alpha$-crystallins, consisting of $\alpha \mathrm{A}$ and $\alpha \mathrm{B}$ subunits, are members of the small heat shock protein family and have chaperon-like activity, that prevents the aggregation of denatured proteins or the inflammatory reaction in vivo. $\alpha$-crystallins were observed in endotoxin-induced uveitis [30,34]. According to the study of comparative proteome analysis of the human vitreous $[35,36]$, there are $\alpha$-crystallin, which is 
not major protein in the vitreous.

It is suggested that the liquefaction is induced by detachment of non-collagenous protein crystallin beads, resulting in the collapse of the structure to release watery liquid trapped within the meshwork. This is another gelsol transition model for liquefaction.

\subsection{Model of the Structure of Vitreous Body Meshwork Fiber and the Gel-Sol Transition of Liquefaction in Vitreous Body}

It is proposed the models for vitreous body in Figures $\mathbf{5}$ and $\mathbf{6}$ to elucidate the structure of vitreous body and liquefaction process. The model in Figure 5 shows the fiber structure of vitreous body. The surface is coated with Hyaluronic acid and the center core of fiber is collagen with bead of crystallin. In liquefaction, the core collagen may be exposed outside by deforming this fine structure. The model in Figure 6 shows the normal gel-sol transition model (a) and the gel-sol transition model of vitreous body (b). The gel-sol transition model of vitreous body means that it is the irreversible process and the conformational change induces fiber aggregates and the release of watery liquid and crystallins. This gel-sol transition of vitreous body in Figure 6(b) is very different from the normal gel-sol transition of polymer as shown in Figure 6(a).

Further researches and studies are necessary for this more precise elucidation.

\section{ACKNOWLEDGEMENTS}

We acknowledge the late Dr. A. Yamauchi for his continuous encouragement to this research. One of the authors (Kodama) thanks M. Hayashi, M. Ishii, M. Ogiso and N. Fujii for their experimental assists.

\section{REFERENCES}

[1] Sebag, J. (2009) Vitreous: The resplendent enigma. British Journal of Ophthalmology, 93, 989-991. doi:10.1136/bjo.2009.157313

[2] Berman, E.R. (1991) Vitreous. In: Graymore, C.N., Ed., Biochemistry of the Eye, Plenum Press, New York, 291307

[3] Balazs, E.A. (1961) Molecular morphology of the vitreous body. In: Smelser, G.K., Ed., The Structure of the Eye, Academic Press, New York, 293-310.

[4] Davison, P.F. and Seery, C.M. (1993) An analysis of two fibril systems in the mammalian vitreous. Current Eye Research, 12, 107-114 doi:10.3109/02713689308999478

[5] Worst, J.G.F. and Losm L.I. (1999) Cisternal anatomy of the vitreous. Kugler Publications, Amsterdam \& New York.

[6] Brewton, R.G. and Mayne, R. (1992) Mammalian vitreous humor contains networks of hyaluronan molecules. Experimental Cell Research, 198, 237-249.
doi:10.1016/0014-4827(92)90376-J

[7] Sebag, J. and Balazs, E.A. (1985) Human vitreous fibers and vitreoretinal disease. Transaction of the Ophthalmological Society of the United Kingdom, 104, 123-127. http://highwire.stanford.edu/cgi/medline/pmid;3857770? $\underline{\text { maxto }}$

[8] Kodama, M., Hayashi, M., Ishii, M., Fujii, N., Matsuura, T. and Hara, Y. (2010) Structure of vitreous body-Approach to liquefaction using animal model. Folia Japonica de Ophthalmologica Clinica (in Japanese), 3, 10941100 .

[9] Kobayashi, A. (1992) The preparation method for dog eye ball specimen. Jikken Byorisosiki Gijyutu Kenkyu Kaishi (in Japanese), 1, 8-102.

[10] Mallory, F.B. (1938) Pathological technique. W. B. Saunders, Philadelphia.

[11] Mowry, R.W. (1963) The special value of methods that color both acidic and vicinal hydroxyl groups in the histochemical study of mucins withrevised directions for the colloidal iron stain, the use of alcian blue G8X and their combinations with the periodic acid-schiff reaction. Annals of the New York Academy of Sciences, 402-423.

[12] Curtis, F. (1905) Methode de colorationelective du tissue conjonctif. C.R. soc. Biol., 58, 1038-1040.

[13] Asakura, A. (1985) Histochemistry of hyaluronic acid of the bovine vitreous body as studied by electron microscopy. Acta Society of Ophthalmology of Japan, 89, 179191

[14] Scott, J.E. (1992) The chemical morphology of the vitreous. Eye, 6, 553-555. doi:10.1038/eye.1992.120

[15] Bishop, P.N., Holmes, D.F., Kadler, K.E., et al. (2004) Age-related changes on the surface of vitreous collagen fibrils. Investigative Ophthalmology and Visual Science, 45, 1041-1046. doi:10.1167/iovs.03-1017

[16] Los, L.I., van der Worp, R.J., van Luynm, M.J.A., et al. (2003) Age-related liquefaction of the human vitreous body: LM and TEM evaluation of the role of proteoglycans and collagen. Investigative Ophthalmology and Visual Science, 44, 2828-2833. doi:10.1167/iovs.02-0588

[17] van Deemer, M., Pas, H.H., Kuijer, R., et al. (2009) Enzymatic breakdown of type 2 collagen in the human vitreous. Investigative Ophthalmology and Visual Science, 50, 4552-4560. doi:10.1167/iovs.08-3125

[18] Lovicu, F.J., Chamberlain, C.G. and McAvoy, J.W. (1995) Differential effects of aqueous and vitreous on fiber differentiation and extracellular matrix accumulation in lens epithelial explants. Investigative Ophthalmology and Visual Science, 36, 1459-1469.

[19] Ponsioen, T.L., van Deemer, M., Bank, R.A., et al. (2009) Mature enzymatic collagen cross-links, hydroxylsylpyridinoline and lysylpyridinoline, in the aging human vitreous. Investigative Ophthalmology and Visual Science, 50, 1041-1045. doi:10.1167/iovs.08-1714

[20] Grandofer, A. (2008) Enzymatic vitreous disruption. Eye, 22, 1273-1279. doi:10.1038/eye.2008.29

[21] Hikichi, T., Ueno, N., Chakrabarti, B. and Trempe, C.L. (1996) Vitreous changes during ocular inflammation induced by interleukin 13. Japanese Journal of Ophthal- 
mology, 40, 297-302

[22] Akiba, J., Ueno, N. and Chakrabarti, B. (1994) Mechanism of photo-induced vitreous liquefaction. Current Eye Research, 13, 505-512. doi:10.3109/02713689408999882

[23] Ponsioen, T.L., Hooymans, J.M.M. and Los, L.I. (2010) Remodelling of the human vitreous and vitreoretinal interface-A dynamic Process. Progress Retinal Eye Research, 29, 580-595. doi:10.1016/j.preteyeres.2010.07.001

[24] Nishikawa, S. and Tamai, M. (1996) Ultrastructure of hyaluronic acid and collagen in the human vitreous. Current Eye Research, 15, 37-43. doi:10.3109/02713689609017609

[25] Balazs, E.A. and Denlinger, J.L. (1984) The vitreous. In: Davison, Ed., The Eye, Academic Press, London, 533589.

[26] Fujii, N., Satoh, K.H. and Ishibashi, Y. (1994) Simultaneous stereoinversion and isomerization at specific aspartic acid residues in a A-crystallin from human lens. Journal of Biochemistry, 116, 663-669.

[27] Pal, J., Bera, S. and Ghosh, S.K. (1999) Acetylation of $\alpha$ crystallin with $\mathrm{N}$-acetylimidazole and its influence upon the native aggregate and subunit reassembly. Current Eye Research, 19, 358-367.

[28] Srinivasan, A.N., Nagineni, C.N. and Bhat, S.P. (1992) A-crystallin is expressed in non-ocular tissues. Journal of Biological Chemistry, 267, 23337-23342

[29] Yamane, K., Minamoto, A., Yamashita, H., Takamura, H., Miyamoto-Myoken, Y., Yoshizato, K., Nabetani, T., Tsugita, A. and Mishima, H. (2003) Proteome analysis of human vitreous proteins. Molecular \& Cellular Proteomics, 2, 1177-1187.

[30] Bahk, S.C., Jang, J.U., Chai, C.U., Lee, S.H., Park, Z.Y.,
Yang, J.Y., Kim, J.D., Yang, Y.S. and Chung, H.T. (2007) Post-translational modeification of crystallins in vitreous body from experimental autoimmune uveitis of rats. Journal of Proteome Research, 6, 3891-3898. doi:10.1021/pr070133k

[31] Tamburro, D., Facchiano, F., Petricoin, E.F., Liotta, L.A. and Zhou, W. (2010) Mass spectrometry-based characterization of the vitreous phophoproteome. ProteomicsClinical Application, 4, 839-846.

[32] Linklater, H.A., Dzialoszynski, T., MaLeod, H.L., Sanford, S.E. and Trevitrhick, J.R. (1992) Modeling cortical cataractogenesis XII: Supplemental vitamin a treatment reduces gamma crystallin leakage from lenses in diabetic rats. Lens and Eye Toxicity Research, 9, 115-126.

[33] Lutyen-Drecoll, E., May, C.A., Polansky, J.R., Johnson, D.H., Bloemendal, H. and Nguyen, T.D. (1998) Localization of the stress proteins y-crystallin and trabecular meshwork inducible glucocorticoid response protein in normal and glaucomatous trabecular meshwork. Investigative Ophthalmology and Visual Science, 39, 517-525.

[34] Imai, H., Ohta, K., Yoshida, A., Suzuki, S., Hashizume, K., Usami, S. and Kikuchi, T. (2010) Crystallin, new candidate proteins in endotoxin-induced uveitis. Investigative Ophthalmology and Visual Science, 51, 3554. doi:10.1167/iovs.09-3728

[35] Feener, E.P., Madigan, M.C., Provis, J.M., Gao, B., Sadun, A.A., Aiello, L.P. and Sebag, J. (2010) Comparative proteome analysis of the developing and adult human vitreous. Investigative Ophthalmology and Visual Science, 51, 5096.

[36] Angi, M., Kalirai, H., Coupland, S.E., et al., (2012) Proteomic analyses of the vitreous humour. Mediations of Inflammation, 2012, 148039. 